Article

\title{
Application of an Automatic Noise or Signal Removal Algorithm Based on Synchrosqueezed Continuous Wavelet Transform of Passive Surface Wave Imaging: A Case Study in Sichuan, China
}

\author{
Jie Fang $(\mathbb{D}$, Guofeng Liu *(1) and Yu Liu \\ School of Geophysics and Information Technology, China University of Geosciences, Beijing 100083, China; \\ fangjie@cugb.edu.cn (J.F.); 3010200013@cugb.edu.cn (Y.L.) \\ * Correspondence: liugf@cugb.edu.cn
}

check for

updates

Citation: Fang, J.; Liu, G.; Liu, Y. Application of an Automatic Noise or Signal Removal Algorithm Based on Synchrosqueezed Continuous Wavelet Transform of Passive Surface Wave Imaging: A Case Study in Sichuan, China. Appl. Sci. 2021, 11, 11718. https://doi.org/10.3390/ app112411718

Academic Editor: Amerigo Capria

Received: 6 October 2021

Accepted: 7 December 2021

Published: 9 December 2021

Publisher's Note: MDPI stays neutral with regard to jurisdictional claims in published maps and institutional affiliations.

Copyright: (c) 2021 by the authors. Licensee MDPI, Basel, Switzerland. This article is an open access article distributed under the terms and conditions of the Creative Commons Attribution (CC BY) license (https:/ / creativecommons.org/licenses/by/ $4.0 /)$.

\begin{abstract}
Passive surface wave imaging based on noise cross-correlation has been a research hotspot in recent years. However, because randomness of noise is difficult to achieve in reality, prominent noise sources will inevitably affect the dispersion measurement. Additionally, in order to recover high-fidelity surface waves, the time series input during cross-correlation calculation is usually very long, which greatly limits the efficiency of passive surface wave imaging. With an automatic noise or signal removal algorithm based on synchrosqueezed continuous wavelet transform (SS-CWT), these problems can be alleviated. We applied this method to 1-h passive datasets acquired in Sichuan province, China; separated the prominent noise events in the raw field data, and enhanced the cross-correlation reconstructed surface waves, effectively improving the accuracy of the dispersion measurement. Then, using the conventional surface wave inversion method, the shear wave velocity profile of the underground structure in this area was obtained.
\end{abstract}

Keywords: passive seismic; noise separation; surface wave; dispersion curve

\section{Introduction}

Passive seismic methods do not require the excitation of explosives, vibroseis, etc.; they simply require the placement of node geophones according to the active source acquisition line and point spacing, and the data can be collected by continuously receiving ambient noises. Then, by using seismic interferometry (SI), the responses of virtual sources can be retrieved through the correlated responses on the two receivers [1-4]. The term "interferometry" generally refers to the study of interference between signals in order to obtain information through the differences between them. In the field of SI, it is used to study the interference of seismic-related signals. Its basic operation steps are very simple, mainly divided into two steps: the first step is cross-correlation calculation, which can be understood as detecting the travel time difference of waves recorded between two receivers; the second step is stacking-that is, integrating all actual sources.

Although the theory of SI is not limited to either body or surface waves [5,6], extracting surface waves from the cross-correlation of ambient noise is quite robust and has become a mature technology [7]. In contrast, it is much more difficult to extract body waves $[8,9]$. However, some studies have also shown its possibility at various scales [10-13]. Even so, in most SI studies, retrieving surface waves is still one of the major applications. Surface waves with different periods can be used to investigate the earth structure at different depths [14]. Generally speaking, most studies focus on frequencies lower than $1 \mathrm{~Hz}$ to provide images of the structure of the crust and upper mantle $[15,16]$. However, some studies have proven that structures tens to hundreds of meters underground can be imaged with frequencies higher than $1 \mathrm{~Hz}$, such as by using traffic noise to detect urban underground space $[17,18]$, which has attracted the attention of more and more engineering seismologists. 
Green's function reconstruction of diffuse wavefield (i.e., waves arrive from all angles with equal strength) observations in an open configuration is the basis for SI with passive data [19]. However, it is not realistic to obtain a pure diffuse wavefield from observations. Non-ideal source distributions such as limited azimuthal distribution or near-source effects [20] can significantly affect the reliability and interpretation of observations due to the violation of the stationarity assumptions required by passive survey methods [21]. In order to improve the accuracy of the dispersion measurement, some studies have begun to consider how to attenuate the influence of noise sources with prominent directivity. Aki [5] proposed a classical spatial autocorrelation method which used the mathematical transformation of a symmetric receiving array (e.g., circle) to eliminate the azimuth factor of the noise source. Park et al. [22] introduced a novel strategy for imaging dispersion of passive surface waves with an active scheme based on phase-shift measurement, called roadside passive multichannel analysis of surface waves. Scanning processes were implemented along potential azimuthal directions of noise. Feuvre et al. [23] proposed a cross-correlation-based beamforming average dispersion analysis method which effectively restrained the directionality of noise and reduced the aliasing effect. Cheng et al. [24] proposed a multichannel analysis of the passive surface (MAPS) wave method based on long noise sequence cross-correlation to handle the azimuthal effects for directional noise sources. Distinct from the above methods, Mousavi et al. [25] proposed a new and fast algorithm for accurate noise removal/signal removal based on higher-order statistics (HOS), general cross-validation (GCV), and wavelet hard thresholding (WHT) in synchrosqueezed domains and tested the performance of the proposed algorithm with synthetic and real seismic data. In addition, he indicated that it can also be an effective procedure in ambient noise studies.

In this paper, we applied the noise or signal removal algorithm based on SS-CWT proposed by Mousavi et al. [25] to a 1-h passive seismic dataset acquired in Sichuan, China, and verified the effectiveness of this method in enhancing passive surface waves from short time series. First, we used this method to preprocess the 1-hour passive seismic data in Sichuan to remove the prominent noise events and used cross-correlation to produce virtual shot gathers from the preprocessed noise records. Then, after cross-correlation, the method was used as a post-processing step to denoise the virtual shot gathers, improving the signalto-noise ratio $(\mathrm{S} / \mathrm{N})$ of the virtual shot gathers and further improving the reconstructed surface waves. Next, after preprocessing and post-processing, virtual shot gathers were used for dispersion analysis with an active scheme based on the phase-shift measurement, and inversion was conducted to obtain the underground shear wave velocity profile in this area. Finally, the applicability and shortcomings of this method are discussed.

\section{Methodology}

The methodology used in this paper was proposed by Mousavi et al. [25]. We summarized and sorted out their methods, mainly including the following three processing steps.

\subsection{Preprocessing}

For the passive seismic data $y$ collected in the field, first, we carried out CWT and calculated its coefficient $W_{y}$, which represents the finite energy of the data in a concentrated time-frequency picture. CWT is a multiresolution transform method given by [26], as shown in Equation (1):

$$
W_{y}(a, \tau)=\left\langle y, \psi_{a, \tau}\right\rangle=\int_{-\infty}^{+\infty} y(t) a^{-\frac{1}{2}} \psi^{*}\left(\frac{t-\tau}{a}\right) d t
$$

where $a$ and $\tau$ denote the scale and time shift, respectively; $*$ is the complex conjugate; $y, \psi$ denotes the inner product; and $\psi$ is the mother wavelet.

Then, we used HOS and the kurtosis criterion to detect the scale of and remove the pure Gaussian noise correlation coefficient from the time-frequency analysis (TFR), leaving 
the combination of noise and signal. The Kurt of $N$ observation coefficients $W_{y}$ can be calculated by Equation (2):

$$
\operatorname{Kurt}_{y}=\frac{\sum_{n=1}^{N}\left(W_{y_{n}-} \mu_{W_{y}}\right)^{4}}{N \sigma_{W_{y}}^{4}}-3,
$$

where $\sigma_{W_{y}}$ and $\mu_{W_{y}}$ are the estimated standard deviation and mean value of wavelet coefficient $W_{y}$, respectively, and $N$ denotes the sample sequence. HOS measure the degree of Gaussianity, so they have been used as detectors of non-Gaussian signals in Gaussian noise [27]. Equation (3) defines the HOS criterion, which is retained for the Gaussianity measure for distinguishing Gaussian distributions from non-Gaussian distributions:

$$
\left|\operatorname{Kurt}_{y}\right| \leq \frac{\sqrt{24 / N}}{\sqrt{1-\alpha}}
$$

where $\alpha$ is the level of confidence. Ravier et al. [28] estimated that the optimal value of $\alpha$ is $90 \%$.

\subsection{Thresholding}

After obtaining the processed coefficient $W_{y}$ through the preprocessing steps, the synchrosqueezed transform was performed according to Equation (4) to obtain the synchrosqueezed-CWT (SS-CWT) coefficients $T_{y}$.

$$
T_{y}\left(\omega_{\ell}, \tau\right)=\Delta \omega^{-1} \sum_{a_{k}\left|\omega\left(a_{k}, \tau\right)-\omega_{\ell}\right| \leq \frac{\Delta \omega}{2}} W_{y}\left(a_{k}, \tau\right) a_{k}{ }^{-\frac{3}{2}} \Delta a_{k},
$$

where $\omega$ is the discrete frequency and $\omega_{\ell}$ represents the $\ell$ th discrete frequency, $a_{k}$ represents the $k$ th scale, $\tau$ is the time shift, and $\Delta$ represents the increment symbol, where $\Delta \mathrm{a}=a_{k}-a_{k-1}$.

Then, we used the hard threshold scheme (Equation (5)) proposed by Donoho et al. [29,30] to provide a threshold for the SS-CWT coefficients $T_{y}$.

$$
\eta_{\lambda}^{h}\left(W_{y}, \lambda\right)= \begin{cases}W_{y} & ,\left|W_{y}\right|>\lambda \\ 0 & ,\left|W_{y}\right|<\lambda\end{cases}
$$

where $\lambda$ is the selected appropriate threshold; $\eta$ is the selected threshold rule, which can be divided into a hard threshold and a soft threshold; $\eta_{\lambda}^{h}$ represents the hard threshold rule; and $W_{y}$ represents the wavelet coefficients of observation $y$.

The optimal threshold $\lambda$ was automatically determined by the GCV method, which was proposed by Nason et al. [31]. The GCV function is defined as follows:

$$
\operatorname{GCV}(\lambda)=\frac{1}{N} \frac{\left\|T_{y}-\widetilde{T}_{\lambda}\right\|^{2}}{\left\|\frac{N_{0}}{N}\right\|^{2}},
$$

where $\widetilde{T_{\lambda}}$ is the threshold coefficient using the threshold $\lambda$, and $N_{0}$ is the number of coefficients using the threshold $\lambda$ to return to zero. This function simulated the error between the estimated signal and the real signal, so its minimum value was used to select an optimal threshold. By selecting thresholds for the main components of the signal, the initial estimation of the signal was obtained using Equation (7):

$$
y_{k}(t)=2 C_{\psi}^{-1} \operatorname{Re}\left(\sum_{l \in L_{k}(t)} T_{y}\left(\omega_{\ell}, t\right)\right),
$$


where $L_{k(t)}$ denotes a small frequency band around the ridge of the $k$ th component in the SS-CWT, the symbol Re represents the real part, and the constant $C_{\psi}$ is given by Thakur et al. [32]:

$$
C_{\psi}=\int_{0}^{\infty} \xi^{-1} \hat{\psi}^{*}(\xi) d \xi
$$

where $*$ represents the complex conjugate; $\psi$ is the mother wavelet, which is a square integrateable function; $\xi$ is the angular frequency; and $\hat{\psi}$ is the Fourier transform of $\psi$.

\subsection{Postprocessing}

In the third step, a simple level-dependent wavelet threshold was applied to the signal obtained in the thresholding steps; the initial estimation of the seismic signal was subjected to CWT, shown in Equation (1); and thresholds of the coefficients of all scales were selected step by step using the hard threshold rule again, as shown in Equation (5). However, the threshold $\lambda$ estimation used here was given by Donoho et al. [29], which was calculated using Equation (9):

$$
\lambda=\sigma_{n} \sqrt{2 \ln N},
$$

where $\sigma_{n}=$ median $\left(\left|W_{y}\right|\right) / 0.6745$. Finally, the signal in the original data was extracted by applying an inverse CWT, as shown in Equation (10):

$$
y(t)=\frac{1}{C_{\psi}} \iint W_{y}(a, \tau) d \tau \frac{d a}{a^{2}} .
$$

For the passive seismic raw records, the extracted signal $y(t)$ represented the records that contained unnecessary prominent noise events, and the uniform background noise could be extracted by subtracting $y(t)$ from the original data $y$.

\section{The Field Data}

The passive seismic data acquisition area is located in a mining area in Sichuan (as shown in the blue square in Figure 1a), where Triassic feldspar quartz sandstone, Jurassic marl-dolomite-feldspar quartz sandstone, Cretaceous purplish red mudstone-siltstonesandstone, and Quaternary strata are exposed. Figure $1 \mathrm{~b}$ shows a diagram of the geological structure of the mining area. The main structural direction of this area is north-northeast, followed by nearly east-west. Magmatic rocks in the mining area are mainly gabbro and diabase from the late Jinning period, which are exposed as rock masses and dikes of different sizes. The wall rock alteration is developed, among which the wall rock alteration related to mineralization mainly includes chloritization, silicification, and carbonization.

We arranged a two-dimensional passive seismic survey line, P18-P18", at the periphery of the mining area (as shown by the red dotted line in Figure $1 \mathrm{~b}$ ). The passive seismic data were continuously recorded for 4 days with a 1-millisecond sample interval using 120 node-type single-component geophone I-Nodal at 1-240 Hz natural frequency. The distance between each geophone was $12.5 \mathrm{~m}$, and the total length of the survey line was $1.5 \mathrm{~km}$. In order to avoid surface wind noise, the geophones were buried at depths of about $20 \mathrm{~cm}$.

The maximum elevation difference of the terrain in the test area was $100 \mathrm{~m}$, and the slope was gentle. The surrounding areas were sparsely populated and underdeveloped, with one or two village-level roads. There were many rivers nearby, and there were mining activities about $4 \mathrm{~km}$ northwest. Comprehensive analysis showed that the noise in this area mainly came from rivers, traffic, and mining activities, and mining activities may be the main source of prominent noise events. Figure 2 shows the daily noise data recorded by a geophone. 


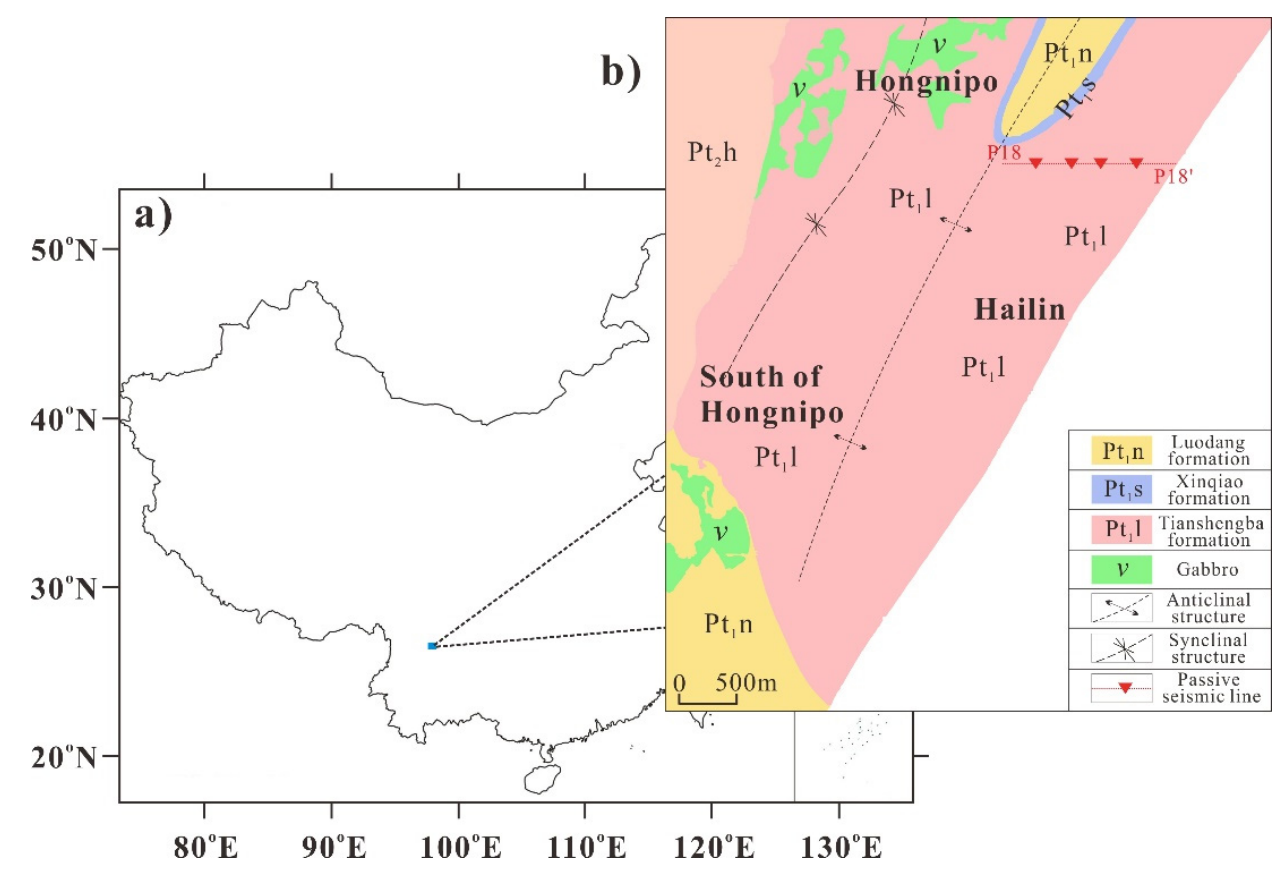

Figure 1. Location of the passive seismic survey line. (a) Location of mining area where data were collected (blue square); (b) geological structure of the mining area. The red dotted line P18-P18' indicates the passive seismic survey line.

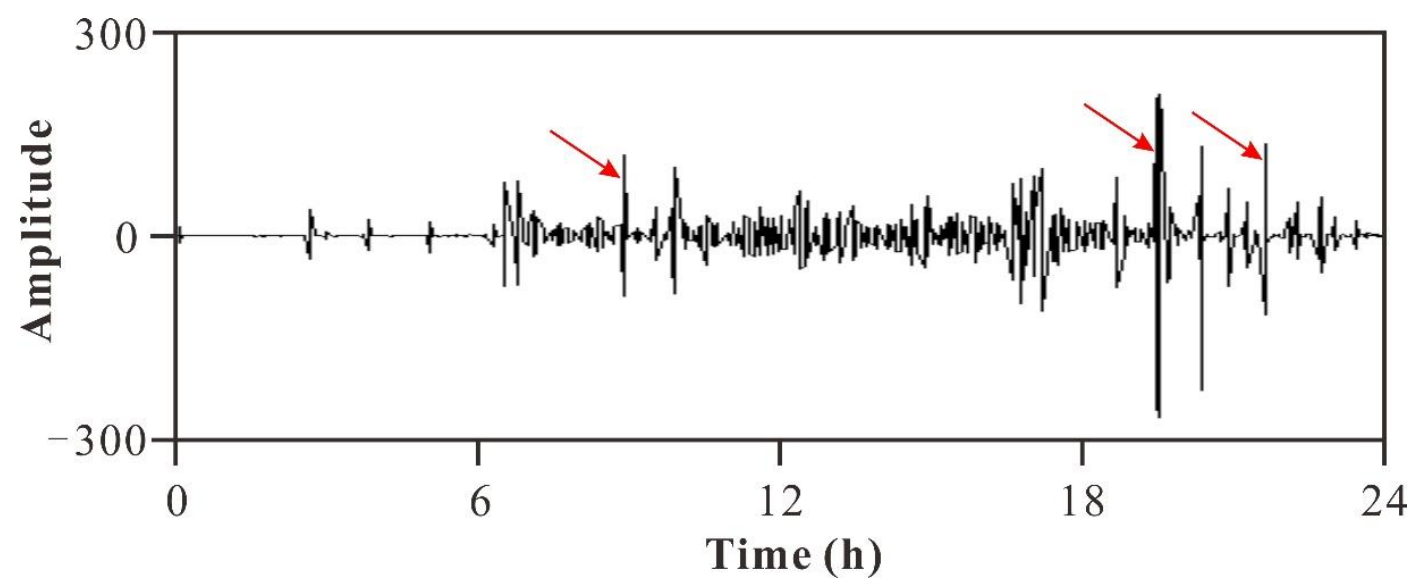

Figure 2. Typical ambient noise data recorded by a geophone in the survey area.

There was very little noise from night to morning $(0-6 \mathrm{~h})$, with relatively low amplitude and stable change, whereas during the day, due to activities such as surface mining, the noise amplitude was relatively high and changed drastically, including some prominent high-amplitude noise events (red arrows). Therefore, in order to satisfy the hypothesis of a theoretical wavefield as much as possible, the original field data were processed.

\section{Field Data Processing}

\subsection{Preprocessing for Raw Field Data}

Raw field data will inevitably be affected by prominent noise events (e.g., mechanical construction), which makes it impossible to satisfy the assumption of a diffuse wavefield. Therefore, in order to reconstruct passive surface waves better, we first used the noise or signal removal algorithm based on SS-CWT proposed by Mousavi et al. [25] to preprocess the field data and separate the prominent noise events. Figure 3a shows an original field record of $90 \mathrm{~s}$ on which several relatively prominent noise events (red arrows) are 
distributed. After noise and signal separation, the amplitude of the separated noise records was relatively evenly distributed (as shown in Figure 3b). Figure 3c shows the removed records containing prominent noise events (red arrows).

(a)

(b)
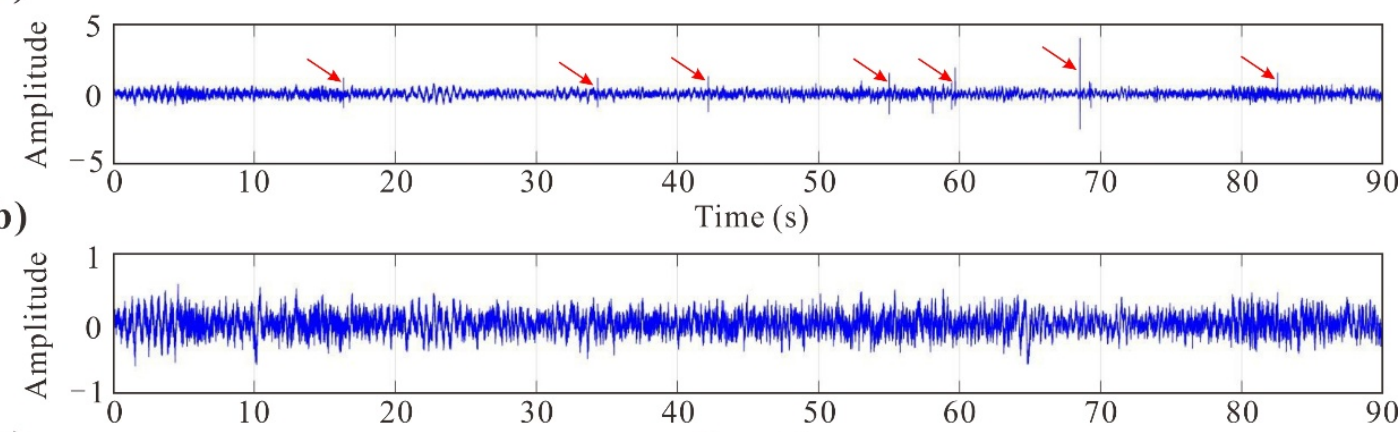

(c)

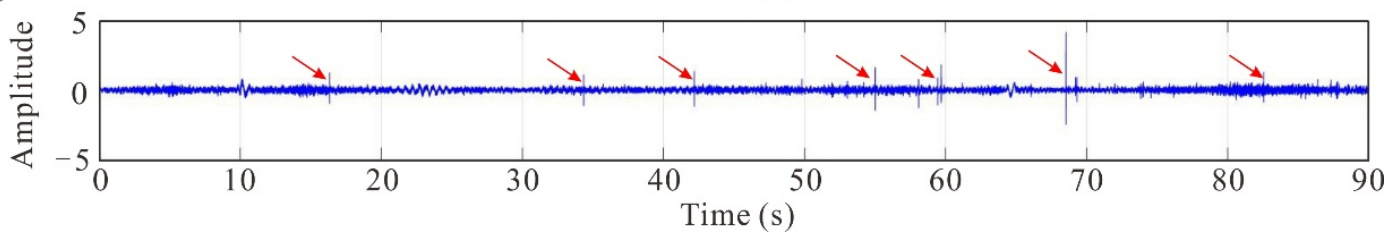

Figure 3. (a) Raw field data of 90 s. (b) Separated noise records. (c) Removed records. The red arrows indicate prominent noise events.

Power spectral density (PSD) plots are useful for visualizing variations in the frequency content of ambient data over time [33]. In order to further show the application effect of the noise separation algorithm in preprocessing the field data, we also calculated the PSD plots (as shown in Figure 4) corresponding to the three noise records in Figure 3.

(a)

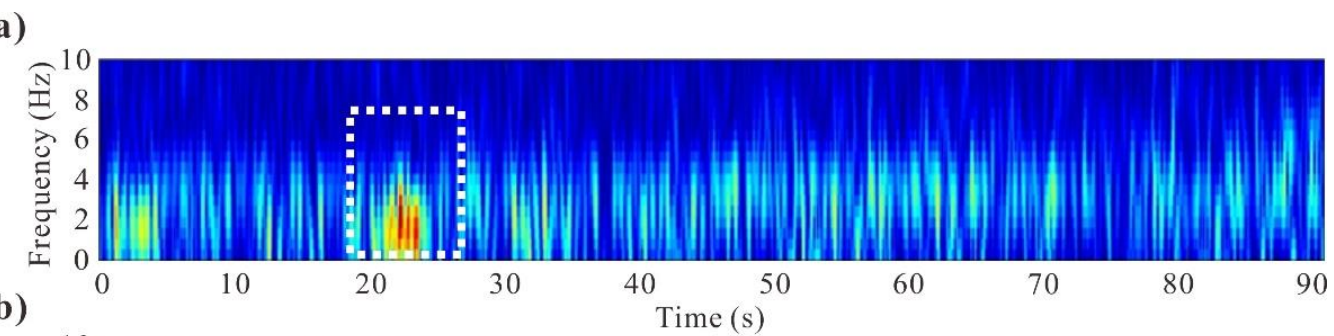

(b)

(c)
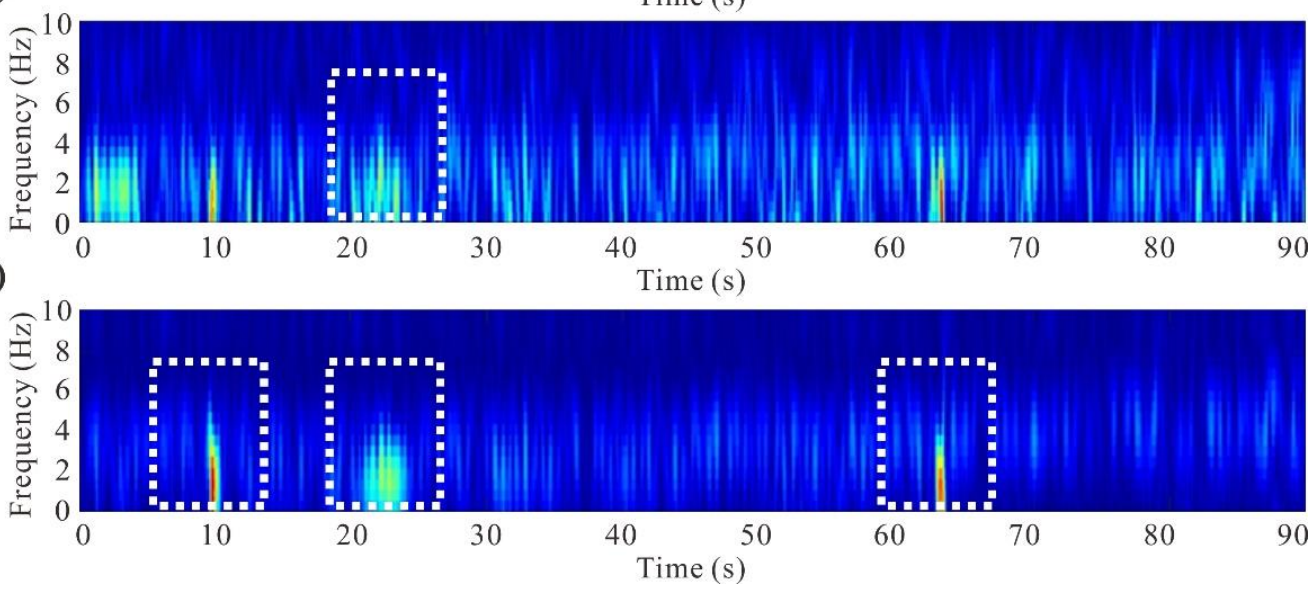

Figure 4. PSD plots. (a) Raw field data. (b) Separated noise records. (c) Removed records. The white dotted boxes indicate prominent noise events.

Figure 4a shows the PSD plots of the original field data, on which a prominent highenergy event (white dotted box) can be seen. After noise separation, as shown in Figure $4 \mathrm{~b}$, 
the high-energy event in the white dotted box was attenuated, and the PSD of the whole record was evenly distributed. Figure $4 \mathrm{c}$ shows the PSD of the removed noise record, which was occupied by three prominent high-energy events (white dashed boxes). According to the results of Figures 3 and 4, the noise or signal removal algorithm based on SS-CWT could effectively remove the prominent noise events in the original passive seismic data.

\subsection{Reconstruction of Virtual Shot Gathers by Cross-Correlation}

After preprocessing the raw field data, one geophone served as a virtual source for waves recorded by other receivers when using the cross-correlation calculation; thus, we could obtain all virtual shot gathers from every receiver without using an active source. In this paper, we showed the virtual shot gathered with the first receiver as the virtual source. In addition, in order to better characterize the intensity of the surface waves on the reconstructed virtual shot gathers, we used the dispersion curve image obtained by the phase-shift measurement method as the visualization tool.

Figure 5a shows the virtual shot gather formed by the cross-correlation of raw passive seismic data acquired in Sichuan for a certain hour. The surface waves (indicated by red arrows) can be seen in the red box on the virtual shot gather but are concealed by the energy of a strong transverse axis. The corresponding dispersion curve image is shown in Figure $5 b$, and the dispersion curve (red part) is disordered and discontinuous.

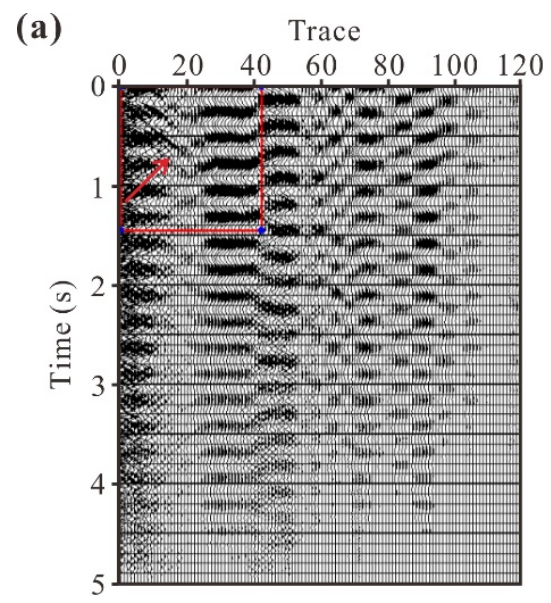

(b)

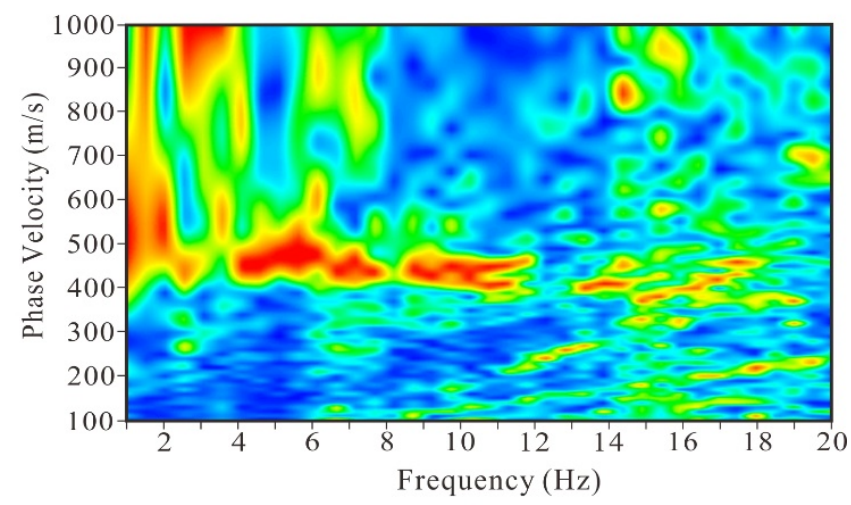

Figure 5. (a) Virtual shot gather formed by cross-correlation of raw one-hour field data and (b) the corresponding dispersion curve image.

After preprocessing the raw field data, the virtual shot gather formed by the separated noise records is shown in Figure 6a. Compared with Figure 5a, the in-phase axis (indicated by the red arrow) of the internal surface waves in the red box for the processed virtual shot gather is clearer, but it is still concealed by the energy of the strong horizontal axis. The corresponding dispersion curve image is shown in Figure 6b, where the curve (red part) is clearer and more continuous than in Figure $5 b$.

By comparing the virtual shot gathers and their corresponding dispersion curve images in Figures 5 and 6, we saw that the virtual shot gather formed by the separated noises had clearer surface wave in-phase axes and dispersion curves, which proved that the noise or signal removal algorithm based on SS-CWT could effectively enhance the reconstructed surface waves after preprocessing the raw field data. 
(a)

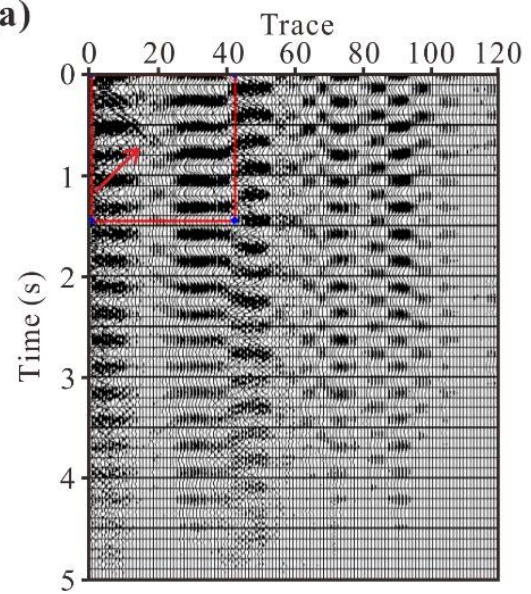

(b)

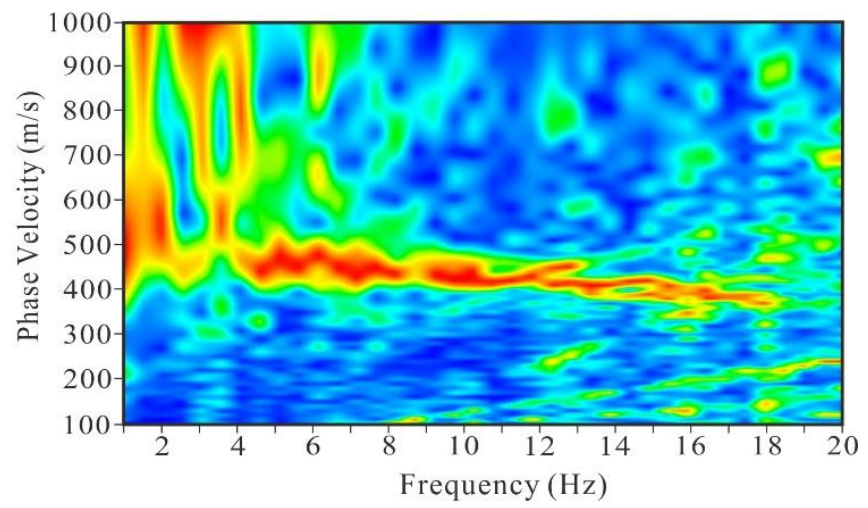

Figure 6. (a) Virtual shot gather formed by cross-correlation of preprocessed separated noises and (b) the corresponding dispersion curve image.

\subsection{Post-Processing for Virtual Shot Gathers}

Although preprocessing the raw field data before cross-correlation could effectively enhance the surface waves, the surface waves on the virtual shot gather were still concealed by the strong transverse axis energy. Determining how to eliminate the strong transverse axis energy, improve the $\mathrm{S} / \mathrm{N}$ of the virtual shot gather, and further enhance the surface waves is of great significance for subsequent inversion and imaging.

Baig et al. [34] proposed several methods to improve the fidelity of noise crosscorrelation based on the discrete orthogonal S transform, which showed that time-frequency denoising of correlograms (i.e., virtual shot gathers) can alleviate this problem. The noise or signal removal algorithm based on SS-CWT proposed by Mousavi et al. [25] can also be applied to the virtual shot gather after cross-correlation to denoise correlograms and improve the S/N. Figure 7 shows the virtual shot gather (Figure 7a) and its dispersion curve image (Figure $7 \mathrm{~b}$ ) after denoising the correlogram of Figure $5 \mathrm{a}$ using the noise or signal removal algorithm based on SS-CWT. Comparing Figure 7a with Figure 5a, the strong transverse axis energy on the correlogram was effectively eliminated, and the surface waves were highlighted. The dispersion curve in Figure $7 \mathrm{~b}$ (red part) is more concentrated and continuous than that in Figure 5b.

(a)

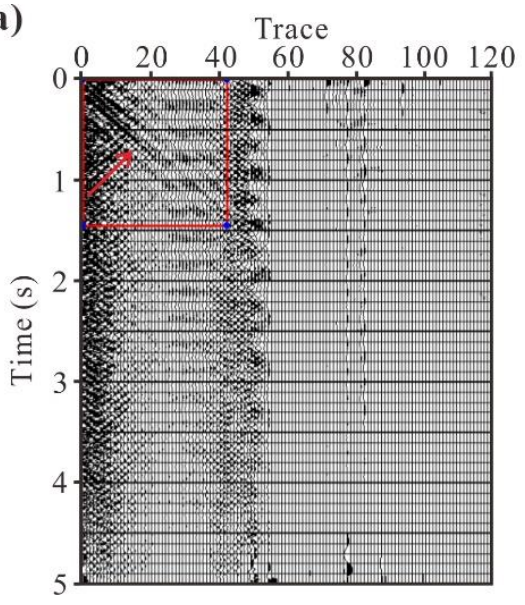

(b)

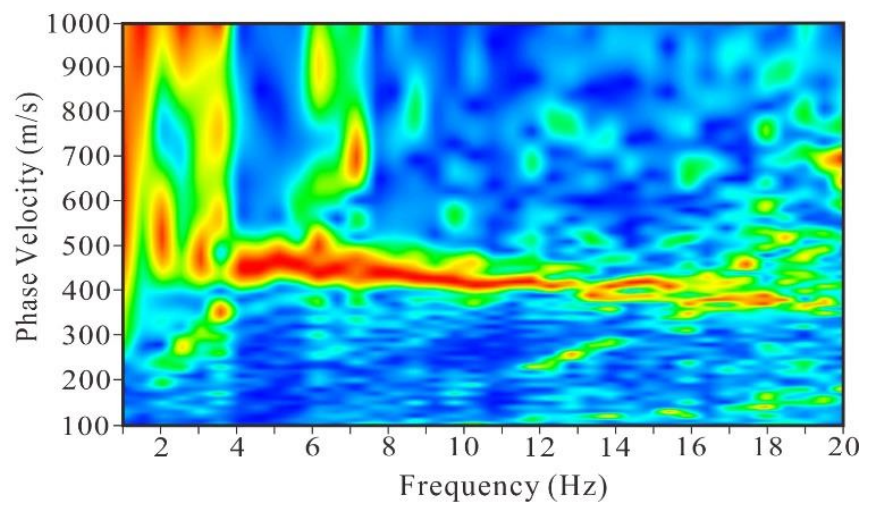

Figure 7. (a) Virtual shot gather after denoising the correlogram of Figure 5a and (b) the corresponding dispersion curve image.

The above research showed that the noise or signal removal algorithm based on SS-CWT could effectively enhance the surface waves after preprocessing the raw field 
data. At the same time, the method could also denoise the correlogram to further enhance the surface waves. Therefore, for the passive seismic datasets acquired in Sichuan, we first preprocessed the raw field data, then used the separated noise records to perform cross-correlation calculations to form the virtual shot gathers, and finally post-processed the correlograms, thus obtaining the final virtual shot gather, shown in Figure 8a, and its corresponding dispersion curve image (Figure 8b).

(a)

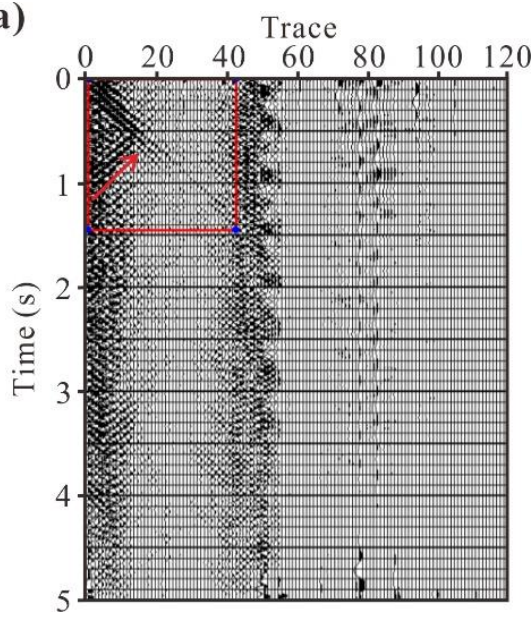

(b)

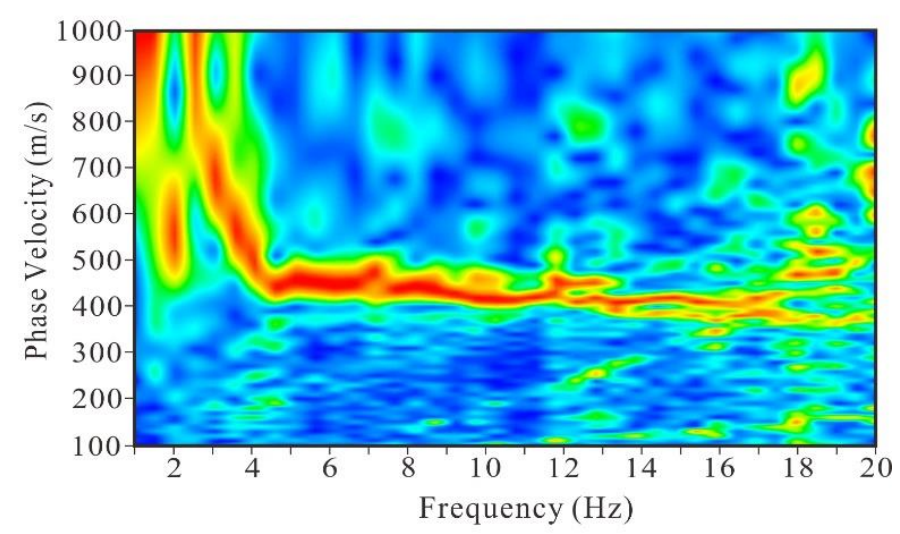

Figure 8. (a) Virtual shot gather after preprocessing and post-processing and (b) the corresponding dispersion curve image.

Comparing Figure 8 with Figures 5-7, we observed that the strong transverse axis energy in the virtual shot gather (Figure 8a) was eliminated, and the surface waves were richer and clearer; the information of the low-frequency part in the dispersion map shown in Figure $8 \mathrm{~b}$ is prominent, and the dispersion curve is clear and continuous.

\subsection{Shear Wave Velocity Profile}

According to the pre- and post-processing steps mentioned above, we obtained virtual shot gathers with each receiver as the virtual shot point. We used phase-shift measurement for dispersion analysis and then used the genetic algorithm [35] to invert the underground shear wave velocity structure.

The genetic algorithm is an efficient, parallel, random global optimization search algorithm based on biological genetic mechanisms and natural selection. It searches for the optimal solution by giving a hypothetical solution and simulating the process of natural evolution. This method has been widely promoted and successfully applied in many fields, and it is also a commonly used Rayleigh wave dispersion curve inversion method. The initial model parameters (i.e., hypothetical solution) and the inversion parameters are shown in Table 1.

Table 1. Initial model parameters and the parameters used for inversion by genetic algorithm.

\begin{tabular}{|c|c|c|c|c|c|c|}
\hline \multicolumn{4}{|c|}{ Parameters Used to Constrain the Initial Model } & \multicolumn{3}{|c|}{ Parameters Used for Inversion } \\
\hline Layer Number & $\begin{array}{l}\text { Lower Limit of Shear } \\
\text { Wave Velocity }(\mathrm{m} / \mathrm{s})\end{array}$ & $\begin{array}{l}\text { Upper Limit of Shear } \\
\text { Wave Velocity }(\mathrm{m} / \mathrm{s})\end{array}$ & $\begin{array}{l}\text { Layer Thickness } \\
\text { (m) }\end{array}$ & $\mathrm{Vp} / \mathrm{Vs}$ & $\begin{array}{l}\text { Density } \\
\left(\mathrm{kg} / \mathrm{m}^{3}\right)\end{array}$ & $\begin{array}{l}\text { Number of } \\
\text { Iterations }\end{array}$ \\
\hline 1 & 200 & 400 & 10 & & & \\
\hline 2 & 400 & 500 & 20 & & & \\
\hline 3 & 500 & 600 & 30 & 2 & 2 & 10,000 \\
\hline 4 & 600 & 800 & 50 & & & \\
\hline 5 & 800 & 1000 & 90 & & & \\
\hline
\end{tabular}

In Figure 9, we show the inversion result of the dispersion curve in Figure 8. Figure 9a shows the initial picked dispersion curve (red dotted line) and the inverted dispersion 
curve (blue line), and Figure $9 \mathrm{~b}$ shows the given upper and lower limits of the initial shear wave velocity model (red dotted line) and the inverted underground shear wave velocity structure (blue line). The high-frequency component of the surface wave mainly reflects the shallow underground information, while the low-frequency component mainly reflects the deep underground information. As there were few or no dispersion data recorded by the passive seismic data in the low-frequency range $(1-2.5 \mathrm{~Hz})$, the inversion results of the shallow part (0-200 $\mathrm{m}$ in Figure $9 \mathrm{~b})$ of the shear wave velocity structure are more accurate, while the inversion results of the deep part (200-300 $\mathrm{m}$ in Figure $9 \mathrm{~b}$ ) are not necessarily the same. Therefore, the depth range of the finally inverted shear wave velocity profile is 0-200 $\mathrm{m}$ underground.

(a)

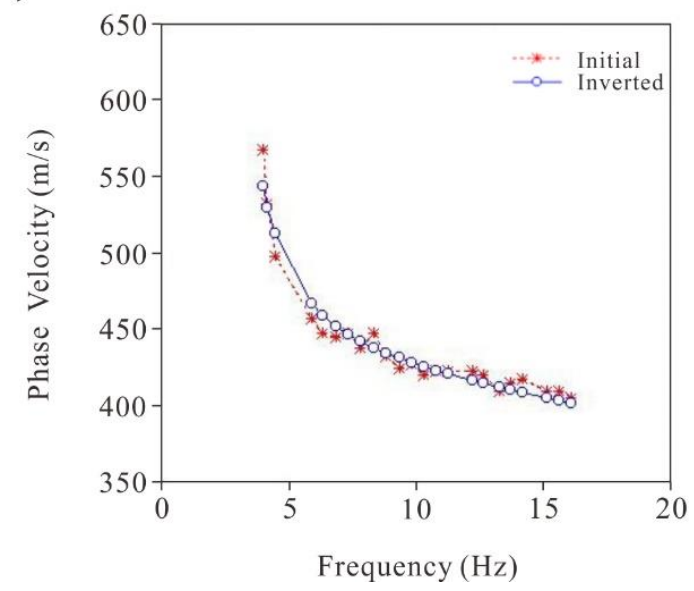

(b)

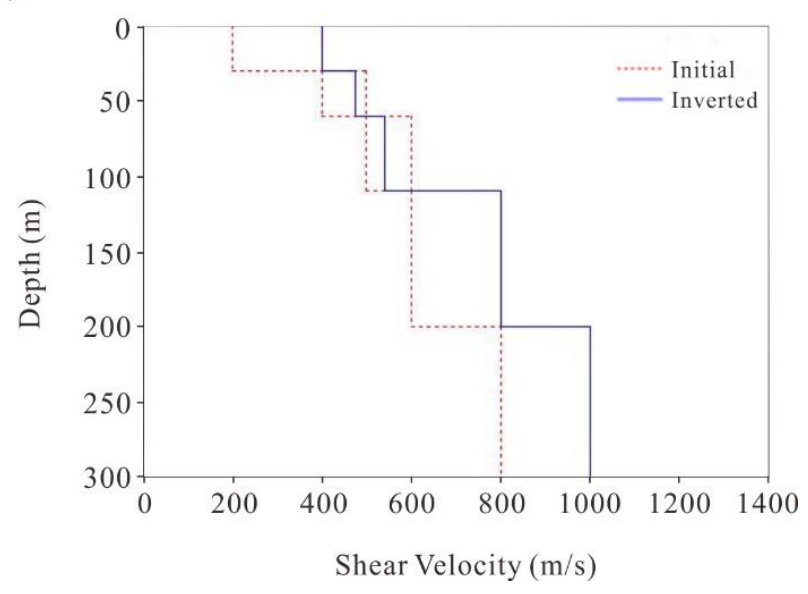

Figure 9. The inversion result of the dispersion curve in Figure 8. (a) The initial picked dispersion curve (red dotted line) and the inverted dispersion curve (blue line). (b) The given upper and lower limits of the initial shear wave velocity model (red dotted line) and inversed shear wave velocity structure (blue line).

We then selected the virtual shot gathers at 30 receivers, used phase-shift measurement for dispersion analysis, and obtained the inverse shear wave velocity structures of those 30 receivers. Finally, the shear wave velocity structure profile in this area was obtained by Kriging interpolation [36], as shown in Figure 10; the red inverted triangle indicates the position of the inverted shear wave velocity structure, and the section length of the final inversion is $1300 \mathrm{~m}$. As the data quality of the following (1300-1500 m) is poor, it cannot avoid bringing errors to the whole inversion, so it was abandoned. Its underground structure is clear, and there is a fault, F, at approximately $900 \mathrm{~m}$.

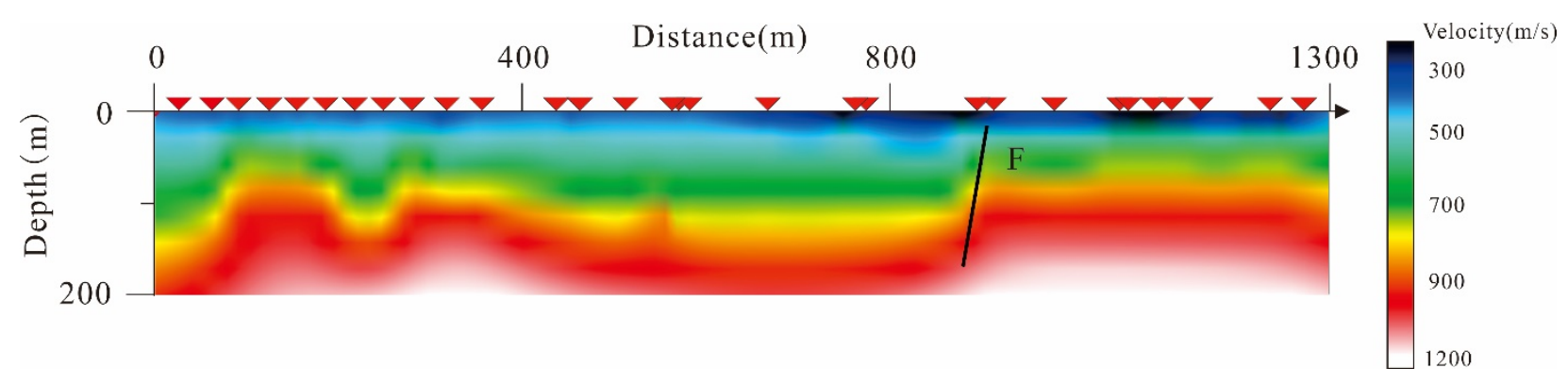

Figure 10. Shallow shear wave velocity profile from passive surface wave inversion.

\section{Discussion}

Although the noise or signal removal algorithm based on SS-CWT proposed by Mousavi et al. [25] has achieved outstanding results in this case study, there are still some problems that need further discussion. In this section, we highlight two issues based on our 
experience of processing the Sichuan passive seismic dataset: (1) the applicability of this method in other passive seismic datasets, and (2) the efficiency of this method in processing passive seismic datasets.

\subsection{The Applicability of This Method in Other Passive Seismic Datasets}

Due to differences in collection environment and conditions, there are often great differences between different passive seismic datasets. Therefore, in addition to testing the passive seismic dataset in Sichuan, we also attempted to apply this method to a passive seismic dataset acquired in Inner Mongolia [37] to enhance the surface waves but did not achieve very obvious results. As the survey line arranged in the research area of Inner Mongolia was located near a village, and it was the busy farming season, when farmers used agricultural machinery to harvest corn in the field, the human activities were complex and the interference was serious, so this method might not be able to completely eliminate these complex noises. However, the study area in this paper is far away from the village, with little human interference, and only the mining activities about $4 \mathrm{~km}$ northwest of the survey line are prominent. Therefore, although this method had a good effect in processing the Sichuan dataset, it may not have obvious effects on the datasets collected in different environments (especially in areas where human activities are prominent).

\subsection{The Efficiency of This Method in Processing Passive Seismic Datasets}

The amount of passive seismic data was relatively large. Taking the Sichuan dataset as an example, the amount of data collected by 120 geophones for $1 \mathrm{~ms}$ was approximately 40 GB a day. When we used this method to process the 1-h data selected by each receiver, it took more than $20 \mathrm{~min}$ to preprocess each trace, and it took $40 \mathrm{~h}$ to complete the whole 1 -h dataset of 120 receivers; thus, the calculation efficiency was relatively low. Therefore, further improvement of this method for calculation efficiency is required before applying it to larger passive seismic datasets.

\section{Conclusions}

We introduced an accurate noise or signal removal algorithm based on SS-CWT. The performance of this algorithm was tested with actual passive seismic data acquired in a certain area of Sichuan, China. The results show that this algorithm can not only eliminate unnecessary prominent coherent noise events in the original passive seismic data but can also denoise the correlogram formed after cross-correlation calculation, improve the S/N of the restored Green function, and enable the reconstruction of a high-fidelity Green function from short time series. However, the computational efficiency of this method is low, and it may not be able to achieve good application results on datasets that are greatly disturbed by human activities.

Author Contributions: Methodology, J.F. and G.L.; validation, J.F., G.L. and Y.L.; writing—original draft preparation, J.F.; writing —review and editing, J.F. and Y.L.; supervision, G.L. All authors have read and agreed to the published version of the manuscript.

Funding: This work was supported by the National Natural Science Foundation of China (grant no. 42074131).

Institutional Review Board Statement: Not applicable.

Informed Consent Statement: Not applicable.

Data Availability Statement: The data presented in this study are available on request from the corresponding author.

Acknowledgments: We thank the editors and four anonymous reviewers for their careful reviews and constructive comments.

Conflicts of Interest: The authors declare no conflict of interest. 


\section{References}

1. Wapenaar, K. Retrieving the elastodynamic Green's function of an arbitrary inhomogeneous medium by crosscorrelation. Phys. Rev. Lett. 2004, 93, 254-301. [CrossRef]

2. Schuster, G.T.; Yu, J.; Sheng, J.; Rickett, J. Interferometric/daylight seismic imaging. Geophys. J. Int. 2004, 157, 838-852. [CrossRef]

3. Weaver, R.L. Information from Seismic Noise. Science 2005, 307, 1568-1569. [CrossRef]

4. Snieder, R.; Şafak, E. Extracting the building response using seismic interferometry: Theory and application to the Millikan library in Pasadena, California. Bull. Seismol. Soc. Am. 2006, 96, 586-598. [CrossRef]

5. Aki, K. Space and time spectra of stationary stochastic waves, with special reference to microtremors. Bull. Earthq. Res. Inst. 1957, 35, 415-456.

6. Claerbout, J.F. Synthesis of a layered medium from its acoustic transmission response. Geophysics 1968, 33, 264-269. [CrossRef]

7. Nakata, N.; Snieder, R.; Tsuji, T.; Larner, K.; Matsuoka, T. Shear-wave imaging from traffic noise using seismic interferometry by cross-coherence. Geophysics 2011, 76, SA97-SA106. [CrossRef]

8. Roux, P.; Sabra, K.G.; Gerstoft, P.; Kuperman, W.A. P-waves from cross-correlation of seismic noise. Geophys. Res. Lett. 2005, 32, 1944-8007. [CrossRef]

9. Forghani, F.; Snieder, R. Underestimation of body waves and feasibility of surface-wave reconstruction by seismic interferometry. Lead. Edge 2010, 29, 790-794. [CrossRef]

10. Draganov, D.; Wapenaar, K.; Mulder, W.; Singer, J.; Verdel, A. Retrieval of reflections from seismic background-noise measurements. Geophys. Res. Lett. 2007, 34, L04305. [CrossRef]

11. Draganov, D.; Campman, X.; Thorbecke, J.; Verdel, A.; Wapenaar, K. Reflection images from ambient seismic noise. Geophysics 2009, 74, A63-A67. [CrossRef]

12. Nakata, N.; Boué, P.; Brenguier, F.; Roux, P.; Ferrazzini, V.; Campillo, M. Body and surface wave reconstruction from seismic noise correlations between arrays at Piton de la Fournaise volcano. Geophys. Res. Lett. 2016, 43, 1047-1054. [CrossRef]

13. Chamarczuk, M.; Malinowski, M.; Draganov, D. 2D body-wave seismic interferometry as a tool for reconnaissance studies and optimization of passive reflection seismic surveys in hardrock environments. J. Appl. Geophys. 2021, 187, 104288. [CrossRef]

14. Cheng, F.; Xia, J.H.; Xu, Y.X.; Xu, Z.B.; Pan, Y.D. A new passive seismic method based on seismic interferometry and multichannel analysis of surface waves. J. Appl. Geophys. 2015, 117, 126-135. [CrossRef]

15. Yang, Y.J.; Ritzwoller, M.H.; Lin, F.C.; Moschetti, M.P.; Shapiro, N.M. Structure of the crust and uppermost mantle beneath the western United States revealed by ambient noise and earthquake tomography. J. Geophys. Res. Solid Earth 2008, 113, B12310. [CrossRef]

16. Guo, Z.; Chen, Y.S.; Yin, W.W. Three-dimensional crustal model of Shanxi graben from 3D joint inversion of ambient noise surface wave and Bouguer gravity anomalies. Chin. J. Geophys. 2015, 58, 821-831. [CrossRef]

17. Behm, M.; Leahy, M.; Snieder, R. Retrieval of local surface wave velocities from traffic noise: An example from the La Barge basin (Wyoming). Geophys. Prospect. 2014, 62, 223-243. [CrossRef]

18. Cheng, F.; Xia, J.H.; Zhang, K.; Zhou, C.J.; Ajo-Franklin, J.B. Phase-weighted slant stacking for surface wave dispersionmeasurement. Geophys. J. Int. 2021, 226, 256-269. [CrossRef]

19. Wapenaar, K.; Draganov, D.; Robertsson, J.O.A. Seismic Interferometry: History and Present Status; Society of Exploration Geophysicists: Tulsa, OK, USA, 2008; pp. 99-101.

20. Roberts, J.; Asten, M. A study of near source effects in arraybased (SPAC) microtremor surveys. Geophys. J. Int. 2008, 174, 159-177. [CrossRef]

21. Claprood, M.; Asten, M.W. Statistical validity control on SPAC microtremor observations recorded with a restricted number of sensors. Bull. Seismol. Soc. Am. 2010, 100, 776-791. [CrossRef]

22. Park, C.B.; Miller, R.D.; Laflen, D.; Neb, C.; Ivanov, J.; Bennett, B.; Huggins, R. Imaging dispersion curves of passive surface waves. In Proceedings of the SEG 74th Annual International Meeting, Denver, CO, USA, 10-15 October 2004; Society of Exploration Geophysicists: Tulsa, OK, USA, 2004; pp. 1357-1360. [CrossRef]

23. Feuvre, M.; Joubert, A.; Leparoux, D.; Côte, P. Passive multi-channel analysis of surface waves with cross-correlations and beamforming: Application to a sea dike. J. Appl. Geophys. 2015, 114, 36-51. [CrossRef]

24. Cheng, F.; Xia, J.H.; Luo, Y.H.; Xu, Z.B.; Wang, L.M.; Shen, C.; Liu, R.F.; Pan, Y.D.; Mi, B.B.; Hu, Y. Multichannel analysis of passive surface waves based on crosscorrelations. Geophysics 2016, 81, EN57-EN66. [CrossRef]

25. Mousavi, S.M.; Langston, C.A. Automatic noise-removal/signal-removal based on general cross-validation thresholding in synchrosqueezed domain and its application on earthquake data. Geophysics 2017, 82, V211-V227. [CrossRef]

26. Mallat, S. A Wavelet Tour of Signal Processing, in Wavelet Analysis and Its Applications, 2nd ed.; Academic Press: Salt Lake City, UT, USA, 1999; p. 620.

27. Swami, A.; Giannakis, G.B. Higher-Order statistics. Signal Process. 1996, 53, 89-91. [CrossRef]

28. Ravier, P.; Amblard, P.O. Wavelet packets and de-noising based on higher-order-statistics for transient detection. Signal Process. 2001, 81, 1909-1926. [CrossRef]

29. Donoho, D.L.; Johnstone, I.M. Ideal spatial adaption by wavelet shrinkage. Biometrika 1994, 81, 425-455. [CrossRef]

30. Donoho, D.L.; Johnstone, I.M. Adapting to unknown smoothness via wavelet shrinkage. J. Am. Stat. Assoc. 1995, 90, 1200-1224. [CrossRef]

31. Nason, G.P. Wavelet shrinkage using cross-validation. J. R. Stat. Soc. Ser. B-Stat. Methodol. 1996, 58, 463-479. [CrossRef] 
32. Thakur, G.; Brevdo, E.; Fučkar, N.S.; Wu, H.T. The Synchrosqueezing algorithm for time-varying spectral analysis: Robustness properties and new paleoclimate applications. Signal Process. 2013, 93, 1079-1094. [CrossRef]

33. Draganov, D.; Campman, X.; Thorbecke, J.; Verdel, A.; Wapenaar, K. Seismic exploration-scale velocities and structure from ambient seismic noise (>1 Hz). J. Geophys. Res. Solid Earth 2013, 118, 4345-4360. [CrossRef]

34. Baig, A.; Campillo, M.; Brenguier, F. Denoising seismic noise cross correlations. J. Geophys. Res. 2009, 114, B08310. [CrossRef]

35. John, H. Genetic Algorithms and the Optimal Allocation of Trials. SIAM J. Comput. 1973, 2, 88-105. [CrossRef]

36. Hansen, R.O. Interpretive gridding by anisotropic kriging. Geophysics 1993, 58, 1491-1497. [CrossRef]

37. Liu, G.F.; Liu, Y.; Meng, X.H.; Liu, L.B.; Su, W.J.; Wang, Y.Z.; Zhang, Z.F. Surface wave and body wave imaging of passive seismic exploration in shallow coverage area application of Inner Mongolia. Chin. J. Geophys. 2021, 64, 937-948. [CrossRef] 